Respiration 2013;85:439-441

DOI: $10.1159 / 000346991$

\section{Description of Free-Flowing Pleural Effusions in Medical Reports after Echographic Assessment}

\author{
Andrea Smargiassi ${ }^{a}$, Riccardo Inchingolo $^{a}$, Alessandro Zanforlin ${ }^{b}$, \\ Salvatore Valente ${ }^{a}$, Gino Soldatic, Giuseppe Maria Corbo ${ }^{a}$, \\ a Pulmonary Medicine Department, University Hospital \\ 'A. Gemelli', Università Cattolica del Sacro Cuore, Rome, \\ b Pulmonary Medicine Unit, General Hospital, Trecenta, and \\ 'Emergency Medicine Unit, Castelnuovo Garfagnana General \\ Hospital, Lucca, Italy
}

The use of chest ultrasonography is now considered a useful diagnostic tool for ever more different pathological conditions [1$3]$. With regards to the echographic assessment of pleural effusions, the usefulness of this technique is well known and globally accepted [2-4].

Pleural effusions can be described on anechoic images above the diaphragm, bordered by the parietal pleural layer at the surface and by the visceral layer at depth, causing lung parenchyma compression, laying in lower areas because of the effect of gravity (with the exception of loculated effusions) [5].

Quantitative assessment of the volume of pleural effusions is crucial to help select patients for thoracentesis. In previous works, many authors have tried to estimate the volume of effusions by measuring the distances between the two pleural layers in different zones of the pleural space, and then compared the measures obtained with the amount of liquid subsequently drained [4-7].

In clinical practice there are pleural effusions that, depending on both the volume and the clinical conditions, should not be drained, but need to be subsequently assessed during medical therapy (i.e. cardiac or renal failure, parapneumonic effusions). Thus, it is crucial to refer to a standardized grading system. However, the echographic description of pleural effusions requires a real-time 'dynamic' medical report which is often dependent on the personal experience and expertise of the operator, and consequently is not completely standardized.

In our opinion, for medical reports dealing with nonloculated pleural effusions which do not have to be drained, estimating the volume and expressing it in milliliters has poor clinical implications because of over/underestimation of volume, which results in difficult computing. In our experience, in fact, the quantitative methods proposed are never applied.

Nowadays, there is not a globally accepted method to classify nonloculated pleural effusions on levels of 'severity' using echographic landmarks, easily checkable and recognizable for everyone, like in the classification methods already accepted for other pathologies (such as chronic obstructive pulmonary disease). We would like to propose an alternative method to standardize medi- cal reports dealing with pleural effusions assessed by chest ultrasonography. The method proposed has the aim of shifting attention from the 'quantification volume method' toward a 'clinical easyway approach'.

In order to propose this method, we tried to describe pleural effusions in 62 consecutive cases evaluated at the Pulmonary Medicine Department of 'A. Gemelli' University Hospital, Rome, Italy. We obtained informed consent from each study participant and the study was approved by the institutional review board.

MyLab $^{\text {TM }} 50$ Cardiovascular (Esaote Spa, Rome, Italy) with a convex 3.5-5 MHz probe was used. The pleural fluid was localized through a longitudinal approach along the posterior axillary line with the patient sitting or lying supine.

Three pneumologists (A.Z., A.S. and R.I.) independently described the same pleural effusion using the new method and each definition required their full concordance, which was achieved in all cases. The landmarks used were:

(1) Anatomic features checked by ultrasound such as the level of compression/atelectasis of the lobes, the evaluation of the dia-

phragm dome and the ability to visualize the pulmonary hilum.

(2) Caudal-cranial extension of the pleural effusion estimated by the number of intercostal spaces through which it was possible to check the fluid.

Using both these landmarks it was possible to create an arbitrary classification method based on the level of 'severity', which was very easy to learn and applicable without subjective interpretations. The standardized grading method is reported in table 1. For each grade proposed, an explicative picture is reported in figure 1. As regards to the 62 cases reported, table 2 shows the characteristics of the pleural effusions and their numeric distribution.

It is evident that extension for three intercostal spaces can equally determine partial as well as full atelectasis of the lower lobe, with or without the ability to see the pulmonary hilum. The extension of fluid over three intercostal spaces seems to be a key level: two different grades (grade 3 and 4 of the classification method) are both possible as this condition could depend on physical characteristics.

As far as the clinical impact of this classification is concerned, the 62 pleural effusions reported underwent immediate pleural procedures (diagnostic thoracentesis, evacuative thoracentesis, pleural drainage) in $68 \%(42 / 62)$ of cases. The decision to perform pleural procedures, however, depended on both the extension (and the general clinical conditions of the patient) and the echographic characteristics of the fluid (highly suggestive of exudative etiology), and it was not directly related to the grade. Grade 2 and 3 pleural effusions did not undergo immediate pleural procedure when

This work was presented as a poster at the Italian Respiratory Congress, Catania, 2012. A.S., R.I. and A.Z. equally contributed to this work.

\section{KARGER}

E-Mail karger@karger.com

www.karger.com/res (c) 2013 S. Karger AG, Base

0025-7931/13/0855-0439\$38.00/0
Riccardo Inchingolo, MD

Pulmonary Medicine Department, Università Cattolica del Sacro Cuore Largo Gemelli, 8

IT-00168 Roma (Italy)

E-Mail r_inchingolo@virgilio.it 
Table 1. Standardized grading method proposed for pleural effusions

\begin{tabular}{llll}
\hline Grading & Description & Landmarks & Intercostal spaces \\
\hline Grade 1: minimum & Limited to costophrenic sinus & Diaphragmatic dome partially evident & $\begin{array}{l}\text { Limited to costophrenic } \\
\text { sinus }\end{array}$ \\
\hline Grade 2: small & $\begin{array}{l}\text { Lower lung lobe partially } \\
\text { involved }\end{array}$ & $\begin{array}{l}\text { Diaphragmatic dome completely } \\
\text { evident }\end{array}$ & 1 intercostal space \\
\hline Grade 3: small to medium & $\begin{array}{l}\text { Lower lung lobe partially } \\
\text { collapsed }\end{array}$ & $\begin{array}{l}\text { Lower lung lobe partially atelectasic } \\
\text { Pulmonary hilum not visible }\end{array}$ & $2-3$ intercostal spaces \\
\hline Grade 4: medium & $\begin{array}{l}\text { Lower lung lobe completely } \\
\text { collapsed }\end{array}$ & $\begin{array}{l}\text { Atelectasis of the lower lung lobe } \\
\text { Pulmonary hilum visible }\end{array}$ & $3-4$ intercostal spaces \\
\hline Grade 5: large & $\begin{array}{l}\text { Upper lung lobe partially } \\
\text { involved }\end{array}$ & $\begin{array}{l}\text { Atelectasis of the lower lung lobe } \\
\text { Upper lung lobe partially atelectasic }\end{array}$ & 4 intercostal spaces or more \\
\hline Grade 6: massive & Lung fully collapsed & $\begin{array}{l}\text { Atelectasis of the whole lung } \\
\text { Hilum completely visible }\end{array}$ & \\
\hline
\end{tabular}

Table 2. Characteristics of the 62 pleural effusions described

\begin{tabular}{|c|c|c|c|c|}
\hline Extension & Features & $\begin{array}{l}\text { Right pleural } \\
\text { effusions, } \mathrm{n}\end{array}$ & $\begin{array}{l}\text { Left pleural } \\
\text { effusions, } \mathrm{n}\end{array}$ & Grade \\
\hline Less than 1 intercostal space & Diaphragmatic dome partially evident & 6 & 3 & 1 \\
\hline 1 intercostal space & $\begin{array}{l}\text { Diaphragmatic dome completely evident } \\
\text { Lower lung lobe partially involved }\end{array}$ & 3 & 4 & 2 \\
\hline 2 intercostal spaces & Lower lung lobe partially atelectasic & 9 & 9 & 3 \\
\hline 3 intercostal spaces & Lower lung lobe partially atelectasic & 6 & 2 & 3 \\
\hline 3 intercostal spaces & $\begin{array}{l}\text { Atelectasis of the lower lung lobe } \\
\text { Pulmonary hilum visible }\end{array}$ & 7 & 2 & 4 \\
\hline 4 intercostal spaces or more & $\begin{array}{l}\text { Atelectasis of the lower lung lobe } \\
\text { Upper lobe eventually involved } \\
\text { Pulmonary hilum visible }\end{array}$ & 6 & 5 & $\geq 4$ \\
\hline
\end{tabular}

they were related to pneumonia or cardiac/renal failure, but they were followed up during medical therapy and possibly downgraded. When upgraded, pleural procedure was then required.

None of the 9 grade 1 pleural effusions immediately underwent pleural procedure, and instead were only followed up. Afterwards, only 1 patient underwent thoracentesis because of subsequent upgrade. Evacuative pleural procedures were performed in all effusions of grade 4 or more due to their extension and their impact on general clinical conditions.

The grading system proposed is crucial in determining whether patients are eligible for pleural procedures when followed up. Moreover, even if a thoracentesis is immediately performed, the grading system is extremely useful for monitoring the same effusion and to guide sequential procedures or medical therapies.

This grading system has some limitations. Firstly it can be applied only to free-flowing pleural effusions since the features of loculated pleural effusions do not match with the criteria here adopted. Secondly, the temporary existence of air in the pleural space, such as in hydropneumothorax, prevents an accurate echographic detection of the landmarks.
In conclusion, these grades determined by the landmarks mentioned, which are well defined and easily identifiable, can contribute to achieving uniformity in the description of free-flowing pleural effusions at ultrasound examination and at 'echographic follow-up' assessment. The necessity to resolve this critical problem in clinical practice deserves to be discussed, which is also the aim of this letter. If this standardized method is taken into account in clinical practice, further studies can be made and the same grading system ameliorated.

\section{Financial Disclosure and Conflicts of Interest}

The authors report that no potential conflicts of interest exist with any companies/organizations whose products or services may be discussed in this article. 


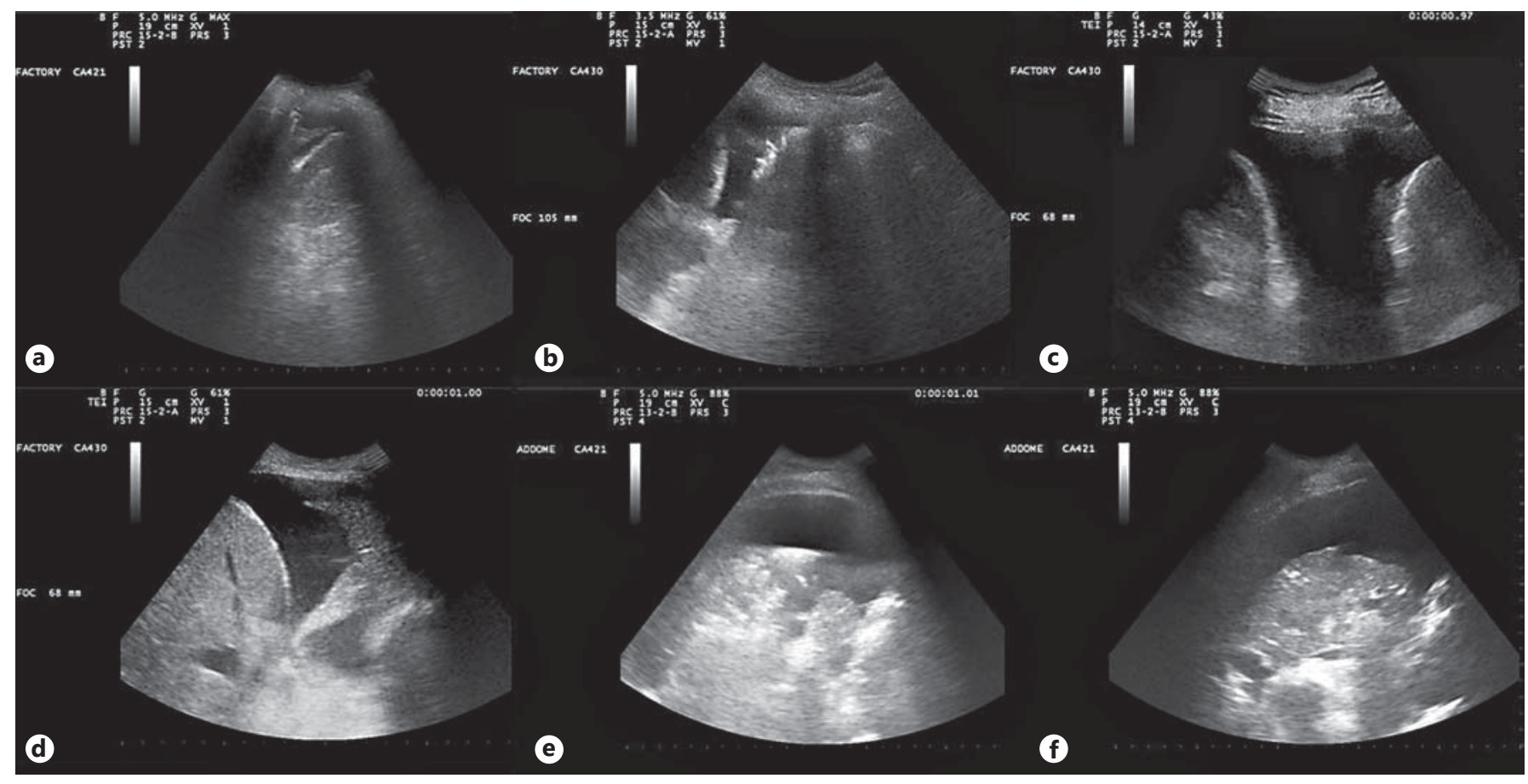

Fig. 1. Explanatory pictures of each grade proposed. a Grade 1: limited to costophrenic sinus with diaphragmatic dome partially evident. b Grade 2: lower lung lobe partially involved with diaphragmatic dome completely evident. c Grade 3: lower lung lobe partially atelectasic with pulmonary hilum not visible. d Grade 4: atelectasis of the lower lung lobe and pulmonary hilum visible. e Grade 5: atelectasis of the lower lung and upper lung lobe partially atelectasic. f Grade 6: atelectasis of the whole lung and hilum completely visible.

\section{References}

-1 Soldati G, Inchingolo R, Smargiassi A, Sher S, Nenna R, Inchingolo CD, Valente S: Ex vivo lung sonography: morphologic-ultrasound relationship. Ultrasound Med Biol 2012;38:1169-1179.

2 Koenig SJ, Narasimhan M, Mayo PH: Thoracic ultrasonography for the pulmonary specialist. Chest 2011;140:1332-1341.

-3 Koegelenberg CFN, von Groote-Bidlingmaier F, Bolliger CT: Transthoracic ultrasonography for the respiratory physician. Respiration 2012;84: 337-350.

-4 Eibenberger KL, Dock WI, Ammann ME, Dorffner R, Hörmann MF, Grabenwöger F: Quantification of pleural effusions: sonography versus radiography. Radiology 1994;191:681-684.
5 Roch A, Bojan M, Michelet P, Romain F, Bregeon F, Papazian L, Auffray JP: Usefulness of ultrasonography in predicting pleural effusions $>500$ $\mathrm{ml}$ in patients receiving mechanical ventilation. Chest 2005;127:224232.

6 Balik M, Plasil P, Waldauf P, Pazout J, Fric M, Otahal M, Pachl J: Ultrasound estimation of volume of pleural fluid in mechanically ventilated patients. Intensive Care Med 2006;32:318-321.

7 Vignon P, Chastagner C, Berkane V, Chardac E, François B, Normand S, Bonnivard M, Clavel M, Pichon N, Preux PM, Maubon A, Gastinne $\mathrm{H}$ : Quantitative assessment of pleural effusion in critically ill patients by means of ultrasonography. Crit Care Med 2005;33:1757-1763. 